\title{
Análisis del Proyecto de Educación Digital (E-DIXGAL): la visión del profesorado de Educación Primaria
}

\author{
Cristina Losada Loureiro \\ cristinalosadaloureiro@edu.xunta.gal \\ Universidad de Santiago de Compostela, España \\ Jesús Rodríguez Rodríguez \\ jesus.rodriguez.rodriguez@usc.es \\ Universidad de Santiago de Compostela, España
}

\begin{abstract}
Resumen
El presente artículo analiza la visión del profesorado sobre el proyecto E-DIXGAL en la Comunidad Autónoma de Galicia. Este proyecto se trata de la primera iniciativa desde la Administración para la implantación del libro digital en los centros educativos. Se toma como referente el proyecto Abalar —denominación autonómica del Programa Escuela 2.0-el cual busca la integración plena de las TIC en la práctica educativa gallega.

El análisis de carácter cualitativo se realiza a través de la técnica de la entrevista aplicada a ocho docentes que pertenecen a centros de carácter urbano y rural de todas las provincias gallegas. Los resultados obtenidos manifiestan un nivel de inclusión de las TIC insuficiente, corroboran los antecedentes teóricos y retratan nuevas problemáticas relacionadas con el desarrollo futuro del proyecto E-DIXGAL.
\end{abstract}

\section{Palabras clave}

Proyecto E-DIXGAL, docentes, TIC, Competencia Digital, aprendizaje en red. 


\title{
Analysis of the Digital Education Project (E-DIXGAL): the views of Primary School teachers
}

\author{
Cristina Losada Loureiro \\ cristinalosadaloureiro@edu.xunta.gal \\ Universidad de Santiago de Compostela \\ Jesús Rodríguez Rodríguez \\ jesus.rodriguez.rodriguez@usc.es \\ Universidad de Santiago de Compostela
}

\begin{abstract}
The following article assesses the vision of teachers about the E-DIXGAL project in the Autonomous Community of Galicia. This project is the first initiative from the Administration for the implementation of the digital book in schools. Thus, it takes as a reference the Abalar project — the autonomic designation of the School 2.0 Program-, which seeks the full integration of ICT in the Galician educational system.

The qualitative analysis is carried out through the technique of the interview applied to eight teachers who belong to urban and rural schools of all the Galician provinces. The results obtained show an insufficient level of ICT inclusion, corroborate the theoretical background and bring new problems related to the future development of the EDIXGAL project.
\end{abstract}

\section{Keywords}

E-DIXGAL project, teachers, ICT, Digital Competence, E-learning. 


\section{Introducción}

Actualmente la influencia de la tecnología es cada vez más notoria, los cambios afectan a la organización del conocimiento, prácticas o incluso a la formación de la propia identidad personal (Adell, 1997; Cabero Almenara, 2015). En efecto, la capacidad para saber utilizar la red es cada vez más importante en la participación de la vida social, política y económica.

La tecnología ha llegado al ámbito escolar en forma de planes y proyectos. La actual Ley de Educación establece siete competencias básicas dentro de las cuales se encuentra la Competencia Digital (CD), desarrollada en el Decreto 105/2014, del 4 de septiembre, que establece el currículo de Educación Primaria en la Comunidad Autónoma de Galicia. La Administración gallega ha apostado por el denominado plan Abalar (2010-2013), que utiliza el modelo 1:1 en las aulas para impulsar un nuevo giro en la pedagogía del aula y desarrollar más fructíferamente la Competencia Digital mencionada. A partir del curso 2014-2015 los centros adscritos a Abalar pudieron participar en el proyecto E-DIXGAL que, usando los mismos equipos de Abalar, supone la digitalización del libro de texto.

Este trabajo pretende analizar el proyecto E-DIXGAL desde la visión del profesorado de Educación Primaria durante el curso 2016-2017. Se analizan y valoran sus objetivos, los cambios que experimentaron desde el inicio y las modificaciones de la cultura y organización escolar después de su incorporación inicial. Valiéndonos de la técnica de la entrevista en profundidad pretendemos comprender y reflexionar sobre los resultados obtenidos con el fin último de lograr un mayor entendimiento del aprendizaje en entornos digitales.

\section{Políticas TIC y libro de texto digital}

Antes de profundizar en el Proyecto E-DIXGAL conviene indagar sobre sus raíces. En el período de transición de las últimas décadas -desde la sociedad industrial hacia la sociedad del conocimiento-, la tecnología afecta a aspectos culturales, económicos, de accesibilidad a la información e incluso en el modo de establecer relaciones sociales. En este sentido la juventud se sitúa en la vanguardia de su uso y se podría definir la generación actual de niños/as como una generación digital interactiva (Bringué, Sádaba, y Artopoulos, 2014; Pérez Sanz, 2011).

La finalidad de la escuela no es otra que la de preparar a los discentes para el futuro que les espera. En realidad, lo que se busca con la inclusión tecnológica en los centros educativos es realzar competencias relativas al aprendizaje permanente, conocimiento personal y alfabetización digital en un mundo cada vez más tecnológico. Con la finalidad de responder a estas demandas de modernización escolar y del sistema educativo en su conjunto nace el programa Escuela 2.0.

\section{a. Plan Abalar}

El programa Escuela 2.0 en Galicia adoptó la denominación de Plan Abalar. Se puso en marcha en el año 2010, basado en la transformación de las aulas tradicionales en digitales mediante el uso de artefactos y herramientas que utilizan software libre. Esta iniciativa puede suponer la apertura hacia la innovación técnica y pedagógica en base a la libertad de expresión y creatividad. La implantación del proyecto Abalar se llevó a cabo en tres fases sucesivas, que corresponden con tres cursos académicos, desde el 2010-2011 hasta el 2012-2013. Segundo el balance de AMTEGA realizado en Abril del año 2014, la situación del proyecto coge forma y se expande paulatinamente por toda Galicia. En la actualidad Abalar tiene más presencia en las provincias de A Coruña y Pontevedra (en 214 y 180 centros respectivamente), mientras que en las provincias de Lugo y Ourense la cifra desciende hasta la mitad. 
Sin embargo, a partir del curso 2012-2013 no se volvió a abrir convocatoria a nuevos centros, aunque si se expandió desde $5^{\circ}$ y $6^{\circ}$ de Educación Primaria hasta $1^{\circ}$ y $2^{\circ}$ de Educación Secundaria. Cabe señalar que la primera fase de selección no contó con una convocatoria ni resolución pública, sino que partió de un ofrecimiento a los centros TIC que quisieran adherirse al proyecto (Fraga Varela y Alonso Ferreiro, 2016).

El proyecto se estructura en cuatro ejes de actuación que se resumen en (AMTEGA, 2014): equipamiento áulico, con un ordenador portátil por persona también denominado modelo $1: 1$, un encerado digital interactivo y acceso a la red; formación de docentes, a través de cursos presenciales y/o en línea; contenidos de uso libre en la plataforma; y herramientas para la comunicación, como por ejemplo el propio Espacio Abalar, Redeiras (comunicación entre el profesorado) y Abalar Móvil (comunicación entre los docentes y las familias).

Abalar apostó por una plataforma diseñada en base al software libre con la finalidad de generar libertad de elección de programas y hacer públicas las renovaciones en beneficio de toda la comunidad educativa. Es evidente que en los últimos años ha emergido un discurso oficial que propugna la lenta desaparición de los libros de texto en papel y su substitución por plataformas de contenidos educativos digitales, lo que evidencia que las políticas educativas siguen teniendo como eje central la dotación tecnológica (Area et al., 2014). Igualmente, la presencia de estas tecnologías como apuntan San Martín y Peirats Chacón (2018), intensifica el trabajo del profesorado, le exigen que sea experto en didáctica, en la disciplina y en el manejo de los artefactos tecnológicos.

\section{b. Proyecto E-DIXGAL}

En el contexto mencionado naceE-DIXGAL en el curso 2014-2015, la primera política de implantación del libro digital en los centros educativos gallegos. Busca fomentar el empleo de las aulas Abalar poniendo a disposición de los centros una plataforma virtual de aprendizaje con libros de texto digitales y otros materiales con los que poder desarrollar la totalidad del currículo (Fraga Varela y Alonso Ferreiro, 2016). La política del libro digital no tomó como base la implantación de los centros educativos comunes, sino que estuvo apoyado expresamente en la Red Abalar. Esto supone que se tomó como marco de referencia el discurso de las políticas de transferencia de tecnología, y para situar la realidad es necesario abordar esta política que permitirá comprender sus implicaciones en el sistema educativo (Fraga Varela y Alonso Ferreiro, 2016; Sanabria Mesa, Álvarez Núñez, Peirats Chacón, 2017).

En el caso de E-DIXGAL, los docentes y el alumnado de los centros implicados desarrollan su actividad educativa diaria en un Entorno Virtual de Aprendizaje (EVA) que les permite acceder a los contenidos digitales de dos proveedores ${ }^{1}$, la editorial $A B$ y la empresa de contenidos digitales $B C$, ambas seleccionadas por la Administración a partir de un concurso público.

Los docentes podrán elaborar su propia "mochila digital" seleccionando a través de la plataforma los contenidos formativos y pudiendo incorporar los materiales complementarios que se deseen, así como los contenidos de elaboración propia (Rodríguez Machado, 2015; Fraga Varela y Alonso Ferreiro, 2016). Asimismo, el acceso a la plataforma es posible a través de cualquier equipo con conexión a Internet, siempre que se haga uso de las claves de acceso que serán entregadas al alumnado en el centro. De este modo el alumnado que así lo desee, tendrá la posibilidad de utilizar también en su casa el netbook empleado en la clase (Rodríguez Machado, 2015).

\footnotetext{
${ }^{1}$ AB y BC son denominaciones ficticias, empleadas para mantener el anonimato de las editoriales
} 
Por otro lado, este entorno depende exclusivamente del profesorado que es quien lo diseña, organiza y secuencia, eligiendo los contenidos de las dos editoriales disponibles. El acceso a los libros digitales se presenta en dos modos (online y offline) con una copia local de los contenidos en el propio equipo que habilita al alumnado a seguir trabajando con los libros de texto digitales en el caso de no contar con conexión a Internet desde sus casas (Fraga Varela y Alonso Ferreiro, 2016).

En este contexto, la forma en la que se implantan los contenidos curriculares en los centros escolares mediante el libro de texto-que constituye el material impreso más empleado en las acciones educativas-, parece asentarse en E-DIXGAL, que contribuye a su proceso de legitimación en una era en la que convergen intereses económicos, políticos y culturales diversos.

Como observa Bonafé (2008) sería conveniente analizar la carga simbólica que lleva consigo una forma de seleccionar, ordenar y presentar los contenidos ya que esta práctica se convierte en un proceso político que puede servir para la reproducción de la orden social o la reconstrucción de la misma mediante la crítica y reflexión.

Igualmente, un contenido pasa a ser valioso y legítimo cuando disfruta del aval social, que a su vez determina la validez y la cultura de la sociedad. Así, después de la incorporación progresiva de tecnología en el sistema escolar es cuando comienzan a concentrarse los esfuerzos sobre el tratamiento digital de los contenidos en el ámbito educativo. Como afirman Peirats et al. (2015), el problema ahora no se reduce sólo a hacer el traspase o reproducir los contenidos desde el soporte analógico al digital, consideran que la cuestión es mucho más compleja ya que tiene que ver con los procesos de producción, distribución y acceso al conocimiento así como con los intereses de la industria editorial.

\section{Planteamiento metodológico}

Como hemos mencionado anteriomente, el proyecto E-DIXGAL se ha iniciado cuatro cursos académicos atrás. Al tratarse de un proyecto relativamente reciente los estudios sobre su desarrollo son escasos y puntuales, referentes a colectivos concretos (alumnado, familias o docentes). Baste como muestra las investigaciones de Fraga Varela y Duarte Fernández (2015), Fraga Varela y Alonso Ferreiro (2016), Fernández de Iglesia, Fernández Morante y Cebreiro López (2016) y Montero Mesa, Gewerc Barujel (2013). Sus conclusiones señalan puntos fuertes como el acceso ilimitado a la información y puntos débiles como la relativa competencia de cada docente en materia TIC.

El presente trabajo pretende analizar el impacto del proyecto E-DIXGAL. En vista de lo expuesto y sin ánimo de generalizar los resultados que se obtengan, en los próximos apartados proponemos ahondar en la visión que tiene el profesorado sobre el proyecto. Esto se hace realidad a través de una investigación cualitativa que emplea la entrevista, realizada a ocho tutores y tutoras pertenecientes a todas las provincias de Galicia, atendiendo a los siguientes criterios: fecha de comienzo de E-DIXGAL, grado de uso, localización, carácter rural o urbano, disponibilidad del profesorado y coordinación TIC.

En la siguiente tabla se especifican los centros elegidos: 


\begin{tabular}{|l|l|l|}
\hline A Coruña & CPI VDC & CEIP LF \\
\hline Lugo & CPI DLS & CEIP RC \\
\hline Ourense & CEIP MBC & CEIP CE \\
\hline Pontevedra & CEIP DPS & CEIP SBL \\
\hline
\end{tabular}

Tabla 1. Docentes participantes en base a la localización y carácter del centro Fuente: Elaboración propia.

Los profesores y profesoras participantes pertenecen a centros públicos, de carácter rural y urbano, de todas las provincias gallegas. Se trata de colegios inmersos en los dos tipos de programas que derivan de Escuela 2.0: Abalar y E-DIXGAL. Por motivos de confidencialidad y anonimato los nombres de los participantes no se encuentran disponibles, debido a esto tanto ahora como durante el análisis se identificará cada participante por el nombre del centro y/o código correspondiente.

Se optó por elaborar una entrevista semi-estructurada y abierta estandarizada que parte de una pauta de preguntas con los temas o elementos clave que se pretende investigar. Entre estos podríamos destacar: la experiencia con el proyecto, opinión sobre su estructura, metodología, relación con el libro de texto o valoración general. El tiempo estimado de las entrevistas fue de 60 minutos.

En lo relativo al proceso de aplicación de las entrevistas, siete de ocho fueron realizadas de forma presencial. Por motivos de limitación en el desplazamiento geográfico la entrevista realizada al CEIP MBC se hizo en formato de videoconferencia mediante la aplicación para ordenador Hangouts.

Todas las entrevistas sin excepción fueron grabadas y transcritas con el programa ExpressScribe. De igual modo, con el fin de obtener la máxima información posible sobre el uso en las aulas del Proyecto E-DIXGAL, decidimos distribuir las preguntas en varios bloques con los que conseguimos dar respuesta a las siguientes cuestiones. 


\begin{tabular}{|l|l|}
\hline \multicolumn{1}{|c|}{ Dimensiones } & \multicolumn{1}{c|}{ Categorías definidas } \\
\hline Datos contextuales & $\begin{array}{l}\text { Años trabajados y cursos TIC. } \\
\text { Opinión sobre la formación de E-DIXGAL. }\end{array}$ \\
\hline Experiencia con E-DIXGAL & $\begin{array}{l}\text { Razones de la adscripción al proyecto. } \\
\text { Papel de E-DIXGAL en el Proyecto Educativo (PE) y en el } \\
\text { Selección de los docentes participantes. } \\
\text { Ventajas e inconvenientes. }\end{array}$ \\
\hline Estructura y modelo & $\begin{array}{l}\text { Estructura del portal de E-DIXGAL. } \\
\text { Opinión sobre los contenidos de las editoriales. } \\
\text { Accesibilidad: alumnado, modo offline. } \\
\text { Modelo pedagógico. }\end{array}$ \\
\hline Metodología & $\begin{array}{l}\text { Tipos de actividades realizadas con E-DIXGAL. } \\
\text { Trabajo por competencias: Competencia Digital (CD). }\end{array}$ \\
\hline E-DIXGAL y el libro de \\
texto & $\begin{array}{l}\text { Opinión sobre la digitalización del libro de texto. } \\
\text { Similitudes y/o diferencias con el libro de texto. }\end{array}$ \\
\hline Valoración general & $\begin{array}{l}\text { Grado de satisfacción con el proyecto E-DIXGAL. } \\
\text { Propuestas de mejora. }\end{array}$ \\
\hline
\end{tabular}

Tabla 1. Dimensiones de análisis. Fuente: elaboración propia.

Con la finalidad de clarificar del mejor modo posible la categorización de la información y la presentación de los resultados se adjunta un código a cada centro educativo (inicial de la provincia más inicial del carácter rural o urbano), que se utilizará en lugar del nombre completo.

- Código CPI VC: CR

- Código CPI DLS: LR

- Código CEIP MBC: OR

- Código CEIP DPS: PR

- Código CEIP LF: CU

- Código CEIP RC: LU

- Código CEIP CE: OU

- Código CEIP SBL: PU 


\section{Resultados}

A continuación, se presentarán los resultados atendiendo a las seis dimensiones propuestas en el apartado anterior atendiendo a la información proporcionada por los/as participantes.

\section{a. Dimensión 1: datos contextuales}

El objetivo de esta dimensión consiste en conocer la experiencia laboral de los/as participantes y la opinión sobre los cursos ofrecidos por la Xunta de Galicia sobre E-DIXGAL. Atendemos a dos categorías: Años trabajados y cursos TIC y Opinión sobre la formación E-DIXGAL.

\section{Años trabajados y cursos TIC}

La mayoría del profesorado posee una larga experiencia docente, a excepción del/la participante del CPI DLS, cuya experiencia es limitada, "es mi primer destino y la primera vez que trabajo como

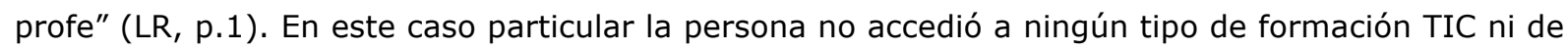
E-DIXGAL.

Por otra parte, los demás participantes afirman haber asistido a cursos de formación del CAFI (Centro Autonómico de Formación e Innovación) de carácter presencial, y también a cursos de formación a distancia en PLATEGA (Plataforma de Teleformación de Galicia) o entidades privadas. Los tópicos de formación más comunes son Ardora, JClic y Realidad Aumentada. Por lo que se refiere a la presencia de cursos presenciales y en línea, los primeros son más comunes y se suelen organizar en el propio centro educativo. El profesorado también se forma a través de la red de forma autodidacta y en parte de los casos por iniciativa propia o curiosidad por probar nuevas herramientas.

\section{Opinión sobre la formación de E-DIXGAL}

El Proyecto E-DIXGAL comenzó en el año escolar 2014-2015, todos los centros participantes de la muestra trabajan con el proyecto desde su implantación y algunos de los/las docentes coinciden en que:

El problema de la formación es que es muy compacta y en poco tiempo, suele ser una formación de 2-3 días, en horario de tarde, algo muy general. No te da tiempo ni a consolidar conocimientos ni a implantarlo en la práctica, normalmente empiezas con EDIXGAL y la formación viene de allí a unos meses. (CR, p.3)

A su vez, indican que "la demanda es muy individualista, es difícil hacer un curso para que esté a nivel de todo el mundo" (OR, p.4), "nosotros nos quejábamos mucho de que no nos separaban por niveles, entonces comienzas siempre por el más básico, y si tú ya sabes lo más básico el curso no lo aprovechas mucho" (PR, p.4-5).

Es necesario mencionar el trabajo individual como principal vía de adquisición de conocimiento por parte de los docentes, por lo que la repercusión de los cursos no es tan positiva como cabría esperar (Fernández Morante y Cebreiro López, 2003). Así mismo, Rodríguez-Machado, Veiga, y González- Sanmamed (2015), entienden que la formación TIC supone un tiempo que va más allá de la labor docente y destacan el carácter autodidacta de la formación, que no se encuentra debidamente reconocida.

Se puede concluir que la formación fue un tanto imprevista, a pesar de ello una parte del profesorado considera que los cursos si fueron de utilidad alegando que les ayudaron a utilizar mejor los recursos de los que disponían. 


\section{b. Dimensión 2: experiencia con E-DIXGAL}

Con esta dimensión se pretende conocer tanto la perspectiva de centro como la individual de cada entrevistado/a a partir de los porqué de la adscripción al proyecto, Papel de E-DIXGAL en el Proyecto Educativo (PE) y en el plan TIC, el proceso de selección del profesorado en el caso de haberlo y, por último, de los puntos fuertes y puntos débiles del proyecto que indican una futura propuesta de mejora.

\section{Razones de la adscripción al proyecto}

Una de las razones para adscribirse se encuentra relacionada con los cambios de la sociedad postindustrial que avanza hacia un modelo de digitalización en todos los ámbitos. Los/as participantes indican que: "necesitamos integrar el uso de las TIC en la vida cotidiana de los centros" ( $C R, p .6)$, "porque evidentemente las nuevas tecnologías son el futuro y no puedes escapar de eso" (OR, p.7).

Algunos de los participantes consideran que adscribirse al proyecto E-DIXGAL significa no quedar atrás en los avances tecnológicos. Se extrae como conclusión la "necesidad" de estar conectados, de contar con ordenadores y con acceso a Internet que permita la accesibilidad a la información en cualquier lugar o momento.

Por otra parte, entre las razones más rompedoras se encuentran las que rechazan sistemáticamente el empleo del libro de texto en las aulas, "no queríamos el libro de texto [...] me cierra mucho el abanico de las cosas que quiero hacer, el primer año no tuve más remedio que aguantarlo porque era lo que había." (LU, p.8). El/La entrevistado/a entiende E-DIXGAL como la superación de la hegemonía del libro de texto en las aulas, sin embargo, es razonable comentar que su visión de la didáctica contempla el trabajo por proyectos que toman como referencia los estándares de aprendizaje del currículo, mezclándolos según la adecuación a cada propuesta de trabajo.

A su vez, también se refleja la visión de E-DIXGAL como la evolución lógica del proyecto Abalar. Mencionar que ambos proyectos optan por el modelo $1: 1$ es importante ya que se sobreentiende como un conductor que garantiza el trabajo con los ordenadores a corto o medio plazo, y permite a los docentes obtener cierta seguridad en el momento de aceptar e incorporarse al proyecto, ya que una de las condiciones consiste en mantener el programa durante un mínimo de dos años.

En definitiva, las razones de la adscripción al proyecto son numerosas y variadas, asimismo, la percepción de E-DIXGAL como la evolución lógica de Abalar cobra importancia a la hora de entrever un hilo que conecte ambos proyectos y ofrezca una razón de peso para seguir trabajando con la tecnología educativa.

\section{Papel de E-DIXGAL en el Proyecto Educativo (PE) y en el Plan TIC}

La misión, visión y valores de un centro educativo quedan explícitos en el PE por lo que sería relativamente lógico que la incorporación de E-DIXGAL transformara sus condiciones de base en los cursos de $5^{\circ}$ y $6^{\circ}$. En este caso la opinión de los/las docentes sobre el proyecto y su papel en el PE y en el Plan TIC es muy diversa, probablemente debido a las particularidades de cada centro.

Para la mayoría de docentes el proyecto sí tiene un papel más o menos relevante: "porque es la base que tenemos" (CU, p.10-11), "tiene un papel relevante en el sentido de que nosotros estamos bien, no nos queremos dar de baja" (LU, p.11), "como otros proyectos, en un mundo 
cada vez más globalizado, quizás posibilita abrir las puertas al mundo y puedes emplear muchos recursos: conexión a la red, herramientas de autor, recursos digitales abiertos, etc." (CR, p. 9). E incluso llegó a propiciar el cambio en la forma de entender los procesos de enseñanza y aprendizaje (E-A) para un/una de los participantes: "a mí me cambió muchas cosas de mi forma de trabajar, no sólo cambié el soporte o dejé emplear libros de texto, sino que empecé a trabajar con redes del tipo Edmodo." (PU, p.12).

Recapitulando, la idiosincrasia de cada escuela juega un papel fundamental en el momento de poner en práctica cualquier proyecto, independientemente de su temática. En referencia la EDIXGAL, en la mayoría de los casos cambió en mayor o menor medida el PE y el Plan TIC.

\section{Selección de los docentes}

En cinco de los ocho centros participantes las personas encargadas de trabajar con E-DIXGAL fueron las que se encontraban en esos cursos cuando salió la convocatoria: "se daba por sentado que el profesorado que estaba trabajando con ese equipamiento en el aula era el que iba a tener E-DIXGAL" (CR, p.12), "el proyecto empezó en $5^{\circ}$ y $6^{\circ}$, entonces la selección que hay es el profesorado que le da, sí o sí." (OR, p.12), "coincidió que estaba yo y otro compañero en $5^{\circ}$ que éramos los que empujábamos un poco para quitar el libro de texto" (LU, p.14).

En estos casos la iniciativa corrió a cargo de un grupo de docentes que llevaron adelante la propuesta y consiguieron que los demás profesores/as se acogieran por solidaridad. En el instante en el que sólo una parte del claustro posee una iniciativa fuerte y decidida se contempla la posibilidad de que surjan más dificultades imprevistas a lo largo del camino, que el/la participante del CEIP MBC expone de forma contundente: "el profesorado que viene nuevo, porque hay mucho interino, cuando llega tiene que cogerlo, no le queda otra. Si está muy a disgusto tiene que aguantarse." (OR, p.12-13). Opinión que corrobora el/la participante del CPI DLS(LR), cuando llegó al centro por primera vez y nunca había escuchado hablar sobre el proyecto. La persona en cuestión debe aprender sobre la marcha, sin ningún tipo de formación más allá de su interés propio y de la que le puedan ofrecer sus compañeros/as.

\section{Ventajas e inconvenientes}

Los/Las entrevistados/as expresaron los puntos fuertes y los puntos débiles del proyecto a partir del contexto en el que se desarrolla, cada uno único. Es necesario recalcar que en algunas entrevistas los/las participantes no diferenciaban entre las ventajas que ofrece E-DIXGAL y las que ofrece un ordenador con acceso a la red, entendiendo el proyecto como este último.

Los puntos fuertes revelan la inmediatez, el rápido acceso a la información y contemplan la posición actual de las familias, reconociendo que es un material gratuito e intuitivo. Esta última cuestión obliga, por otro lado, a abandonar las ayudas para la adquisición de los libros de texto que mencionan Fraga Varela y Alonso Ferreiro (2016), consecuencia natural de utilizar modelos privativos en lugar de recursos más abiertos que permitan la reutilización sin coste.

En contra partida, los puntos débiles del proyecto giran alrededor de dificultades técnicas -que también se sostienen en el trabajo de Rodríguez Machado, Veiga y González Sanmamed (2015) -, la limitación de la actividad docente, la falta de coherencia y cohesión de el proyecto y la visión de las familias como agente educativo poco integrado.

En resumen, las ventajas tienen como común denominador el acceso a Internet, velocidad e inmediatez de la red que permite establecer una dinámica más activa y motivadora. Por su parte, como inconvenientes destacan los problemas de conexión y reparación de equipos, las limitadas opciones editoriales que ofrece E-DIXGAL y la falta de implicación por parte de la comunidad 
educativa.

\section{c. Dimensión 3: estructura y modelo}

El objetivo del análisis de esta dimensión es ahondar en la valoración de los entrevistados/as sobre el proyecto desde la perspectiva de los contenidos que ofrece, herramientas disponibles y el modelo de trabajo que persigue. Así, se pretende acceder a sus creencias y valores más profundos relativos al proceso de Enseñanza y Aprendizaje y E-DIXGAL.

\section{Estructura del portal de E-DIXGAL}

Las opiniones de los/las participantes de la investigación sobre la estructura del proyecto se podrían exponer en dos polos opuestos.

Por un lado, las personas cuya valoración es positiva: "para mí está bien, tú entras en el portal, das de alta el tema que tengas, muy rápido y muy práctico" (OR, p.20), "yo creo que la estructura y la plataforma en sí está bien pensada, cada niño accede con una contraseña y un usuario" (CU, p.21). Tienen en cuenta dimensiones como la facilidad de uso, la cuestión práctica y la seguridad de la plataforma para el alumnado, que ven de manera positiva.

Por otro lado, las personas cuya valoración es negativa centran el foco en aspectos técnicos como por ejemplo el formato de Moodle, que no acaba de encajar para ellos/as y tampoco el formato de E-DIXGAL: "al principio era muy complicado porque no se parece a nada que usara antes" (PR, p.20), "no me gusta mucho, es poco atractivo visualmente para los alumnos y alumnas" (LU, p.21).

En relación a esta categoría las opiniones son muy variadas, incluso contradictorias en función de cada participante. Al margen de esto, el proyecto lleva cuatro cursos académicos funcionando y a pesar del reporte de incidentes a la UAC (Unidad de Atención a Centros), las actuaciones por parte de la Administración son más bien lentas. En este sentido creemos que sería interesante construir un canal de comunicación apropiado que permita a las editoriales y los centros mejorar el proyecto.

\section{Opinión sobre los contenidos de las editoriales}

Como se ha comentado en el segundo apartado, el proyecto E-DIXGAL ha trabajado con dos editoriales que ofrecen contenidos y actividades para desenvolver el currículo de EP, en el curso 2016-2017: AB y BC.

La opinión general del profesorado revela que el repositorio de contenidos es bastante limitado, les gustaría tener más oferta editorial dentro de la plataforma que permitiera adaptar los contenidos a su contexto. En reafirmación, Fraga Varela y Alonso Ferreiro (2016) señalan el impacto de esta situación en la autonomía profesional de los centros educativos y profesorado frente a selección de los materiales educativos. Manifiestan que se trata de un contexto limitador de las posibilidades de creación propia de materiales frente a las propuestas editoriales, sobre las que se espera un uso extensivo debido a la fuerte inversión económica por parte de la Administración, no obstante, no sucede.

Además de no gustar los contenidos, parte de los entrevistados aseguran que el problema se viene arrastrando desde niveles superiores, desde la forma en la que el currículo está diseñado, algunos temas tienen una excesiva carga conceptual que no es fácil abordar en el tiempo de clase.

Para finalizar, sólo queda decir que ninguna de las opciones convence al profesorado, $A B$ por 
reforzar la misma metodología tradicional de corte conductista, y BC por ser incompleta y con contenidos poco apropiados para los/las niños/as de $5^{\circ}$ y $6^{\circ}$ de EP. Quizás se podría tener en cuenta la reflexión de uno de los/las docentes participantes para futuros cambios:

Yo preferiría que se diese una opción pero hecha por gente que lleva mucho tiempo metida en E-DIXGAL y que controla de esto, que sabe que contenidos son buenos... porque en Abalar hay contenidos para trabajar la nutrición, muchísimo mejores que los que tenemos en los libros. (OU, p.27)

\section{Accesibilidad: alumnado, modo offline}

Las preguntas que se refieren a esta categoría suscitaron confusión, la intención era saber si el profesorado creía que el alumnado tenía acceso suficiente a la plataforma, si podían subir contenidos o crearlos en línea, trabajar de forma colaborativa, etc. No obstante, las respuestas se centraron en los siguientes tópicos:

1. La existencia de cierta limitación en el acceso a contenidos: en algunos casos los equipos presentan problemas para cargar correctamente, asimismo, parte del profesorado coincide en que los contenidos sí limitan la práctica docente de algún modo.

2. El escaso uso del modo offline: ninguno de los participantes lo emplea con frecuencia, sólo en el caso de incidentes. Esta situación es posible porque en cinco de los ocho centros participantes los niños/as tienen acceso a la red desde el hogar.

Las diferencias entre las zonas rurales y urbanas de los centros escogidos son significativas, en todos los centros urbanos prácticamente el $100 \%$ del alumnado tiene acceso a internet en el hogar. Sin embargo, en las localidades situadas en zonas rurales, esta cifra se reduce a la mitad. Además, en estas zonas la calidad es bastante inferior a la media y las instalaciones no son suficientes para abastecer a 40 ordenadores -como mínimo- trabajando a la vez. Esto sumado a la dificultad para trabajar desde el hogar en el caso de las zonas rurales posiblemente constituya dos de las razones de más peso para evitar emplear el modo offline.

Llegados a este punto es necesario reflexionar sobre si la necesidad de acceso a internet es real e impuesta indirectamente. La brecha original que expresa Barroso (2013) de una posible fractura social entre los individuos con capacidad para acceder a las herramientas y soportes y los que se verían excluidos sigue en vigencia a raíz de los datos expuestos. A esta brecha se adhiere el riesgo vinculado a la diferencia de capacidad de las personas que dominan las competencias básicas necesarias para construir conocimiento, y las que sólo son usuarios pasivos de las informaciones, en el que influye decisivamente la formación previa de cada docente y familias sobre E-DIXGAL.

\section{Modelo pedagógico}

La finalidad de esta dimensión es establecer una comparación entre lo que el profesorado opina sobre E-DIXGAL y el modelo didáctico que ofrece esta plataforma.

Los resultados muestran que al profesorado le resulta difícil ser objetivo, varios docentes indican que la plataforma ofrece un modelo constructivista, sin embargo, a medida que continúan hablando modifican su opinión. Se observa que estas personas entienden E-DIXGAL como una herramienta que proporciona recursos, actividades y contenidos pero no lo ven como una finalidad en sí mismo. Algunos docentes inciden en una clara inclinación hacia el modelo conductista: "para mí con el transmisivo" (CR, p.33), "en un modelo de transmisión puro y duro" 
(LU, p.35). Y también existe una tendencia a diferenciar las editoriales en función del modelo pedagógico que persiguen, "para mí no tiene nada que ver lo que pretende $\mathrm{BC}$ con lo que inspira $A B$, este último es un libro clásico" (OU, p.34), "hablo de BC, entre cognitivo y constructivista" (PU, p.35). Mientras que $A B$ se sitúa en las teorías conductistas parece ser que BC intenta ir más allá participando de un enfoque más activo e inclusivo.

Sintetizando, la oposición conductista-constructivista se encuentra clara y las valoraciones de la plataforma tienden a asociar el modelo conductista con la editorial $A B$, en tanto que $B C$ es visto como un intento de cambiar el modelo pedagógico recurrente en todos los niveles de la enseñanza obligatoria.

\section{d. Dimensión 4: metodología docente}

El objetivo de esta dimensión es ahondar en la metodología que rige las actuaciones de los/as participantes de la investigación cuando emplean E-DIXGAL. Se hace realidad a partir de las actividades propuestas y su visión sobre el desarrollo de la Compentencia Digital.

\section{Tipos de actividades realizadas con E-DIXGAL}

Los ejercicios más comunes de las editoriales que componen el proyecto según la opinión del profesorado son llenar huecos, asociación o relacionar frases. Constituyen actividades propias de las herramientas de formación TIC que suele recibir el profesorado entrevistado y que se comentaron en categorías anteriores, como por ejemplo Ardora y JClic. Estos ejercicios proporcionan una retroalimentación inmediata aunque no siempre van acompañadas de una explicación en caso de acierto y error. También dan cuenta de pequeñas actividades de investigación que vienen en algunos temas, sin embargo, esto significa una dificultad en el momento de encajarlas en la programación docente debido a la escasez de tiempo para combinar ambas propuestas. Por lo general se aprecia que las actividades y ejercicios contemplados en EDIXGAL no se adaptan a la forma de trabajar de cada profesional.

\section{Trabajo por competencias: Competencia Digital}

Los resultados obtenidos evidencian que los/las participantes de la investigación encuentran dificultades para describir el término Competencia Digital. Las opiniones son diversas e incluso contradictorias entre sí.

Algunos docentes indican que "cuando los niños acaban 50 y $6^{0}$ digitalmente sí que son competentes. Ellos son capaces de buscar información, de elaborar documentos o presentaciones, de subir presentaciones a un aula virtual, de trabajar en un blog." (CU, p.43). Del mismo modo, las comparaciones entre el proyecto EDIXGAL y el software que ofrece el ordenador son constantes a lo largo de todas las entrevistas, las valoraciones positivas se concentran en esta última que supone la posibilidad de elaborar presentaciones, subir contenido a un blog... opciones que en sí mismas no ofrecen las editoriales el proyecto.

Por otro lado, el/la entrevistado/a del CEIP RC opina que el alumnado por lo general no es competente digitalmente: "rotundamente no. Aprenden a darle un ordenador, subir, bajar, poner, quitar, pero eso no es competencia digital."(LU, p.44). Respeto a esto, Rodríguez Machado, Veiga y González Sanmamed (2015) ratifican la opinión con su propia experiencia, en la que el profesorado cree que los alumnos/as no aprenden, sino que se limitan a copiar sin leer y abusan de páginas como Wikipedia para hacer los trabajos en lugar de contrastar la información que reciben. 
En síntesis, la Competencia Digital parece estar adquirida parcialmente (Castro Rodríguez, Marín Suelves y Sáiz Fernández, 2019). El alumnado es capaz de buscar información en la red pero cojea en la parte más importante a desarrollar: la habilidad de seleccionar, contrastar varias fuentes, criticar la información y empezar a desplegar el pensamiento crítico. El reto de la escuela, tal y como indica el/la docente del CEIP SBL, está en "conseguir alfabetizarlos informacionalmente" (PU, p.45).

\section{e. Dimensión 5: E-DIXGAL y el libro de texto}

El análisis de esta dimensión pretende que los/as docentes valoren la conexión entre el Proyecto E-DIXGAL y el libro digital; el libro digital y el libro de texto impreso; y el libro de texto y su papel hegemónico como recurso didáctico.

\section{Opinión sobre la digitalización del libro de texto}

Hasta el curso 2014-2015 los centros adscritos a la red Abalar disponían de los netbook para complementar la metodología de trabajo del profesorado. Con la implantación de E-DIXGAL en los centros Abalar los/as docentes tuvieron que dejar de usar libros de texto como material impreso a favor de los libros digitales.

Para algunos de los entrevistados/as la aparición del libro digital sí significó un cambio significativo que les ayudó a replantear su metodología. Sin embargo, las afirmaciones se contradicen totalmente con la opinión que tienen de las editoriales que conforman el proyecto.

Consecuentemente, la diferencia reside en el empleo del modelo 1:1 como instrumento de referencia en el trabajo diario, cuyo acceso a la red, programas, y herramientas en línea componen la nueva metodología que parece surgir del proyecto. Según los trabajos de Rodríguez Rodríguez, Braga García y Bruillard, (2019) y Rodríguez Rodríguez y Martínez Bonafé (2016), la incorporación de las TIC a las aulas parece no variar ni redefinir el libro de texto en cuanto a objeto y contenido, así lo demuestran ambas editoriales que optan por la separación de contenidos en temas y áreas específicas, aunque el contenido visual sea diferente entre una y otra.

El proceso de adaptación a los equipos fue bastante buena por lo general a pesar de los inconvenientes mencionados en categorías anteriores, relacionados en su mayoría con la presencia de un instrumento nuevo en el aula. Igualmente, el profesorado señala que el trabajo diario no debe quedar exclusivamente en el medio digital, los niños/as tienen que desarrollar la motricidad, jugar con materiales que puedan manipular o crear objetos físicos. Además, consideran que los medios impresos deben seguir formando parte de la actividad diaria, por lo que algunos de los/las entrevistados/as no creen en la digitalización completa de la educación.

\section{Similitudes y/o diferencias con el libro de texto}

Todos y todas las participantes señalan que las diferencias entre E-DIXGAL y el libro de texto son más bien pocas, sólo cambia el soporte, que pasa a ser digital: "el libro está igual ahí" (OR, p.52), "es que yo creo que es el libro de texto en el ordenador" (LR, p.52), "la diferencia es el soporte, que es distinto" (CU, p.52).

Al igual que algunas experiencias no obtienen resultados positivos suficientes, como por ejemplo el estudio de Rodríguez Machado, Veiga y González Sanmamed (2015) en el que ni las TIC ni el Proyecto Abalar aportaron mucho a parte del profesorado, otras experiencias sí los obtienen: los resultados de la investigación de Ballesta Pagano y Martínez Buendía (2015) con el alumnado de 
EP de un centro educativo en Murcia que emplea el libro digital, concluyen que estos sí mejoran en la comprensión de la información, en el desarrollo de una actitud crítica y en la producción informativa.

En resumen, según la visión de los/las profesores/as participantes el Proyecto E-DIXGAL se parece más al libro de texto tradicional que a un nuevo modelo de E-A, a pesar de que BC como se vio en categorías anteriores- supone para ellos y ellas un intento de cambiar el sistema tradicional en el que se encuentran estancados. Las experiencias con el libro digital son diversas y no siempre consiguen buenos resultados, pero utilizadas correctamente pueden suponer un cambio poderoso en la forma de concebir el aprendizaje.

\section{f. Dimensión 6: valoración general}

El objetivo de esta dimensión es analizar el grado de satisfacción general del profesorado con EDIXGAL y las propuestas de mejora sugeridas por los docentes.

\section{Grado de satisfacción con el proyecto E-DIXGAL}

Por lo general el grado de satisfacción es positivo, aunque en algunos casos exige hacer un esfuerzo bastante grande fuera del horario laboral, muchas veces no gratificado. Un/una de los/las participantes reflexiona sobre la comodidad que ofrece el libro tradicional "más eficaz, incluso las editoriales tienen más contenidos, vídeos y hasta los exámenes hechos" (PU, p.56) en comparación con BC -la editorial que emplea él/ella-.

Esto permite establecer las siguientes conclusiones: (1) la importancia del trabajo colaborativo en centros, que permite un reparto del esfuerzo equilibrado entre los docentes. Curiosamente en ninguna categoría los/las entrevistados/as mencionaron los procesos de colaboración como parte fundamental de su trabajo. (2) La relación dual entre el esfuerzo realizado por contextualizar los contenidos y la conmemoración recibida, que no siempre se ve reflejada y acaba por desmotivar al profesorado. (3) La reformulación del papel docente en los procesos de E-A, que pasa de tener el absoluto control de la clase y de las actividades propuestas a tomar el papel de guía y observador participante, siguiendo el modelo constructivista de la enseñanza.

Por otra parte, consideran que el proyecto es útil porque "el uso de las nuevas tecnologías se está utilizando mucho fuera del aula, entonces tenemos que realizar actividades educativas porque sino estamos quedando atrás." (OR, p.55). Es decir, entienden que la digitalización en el ámbito social y económico no puede dejar de lado al educativo. El profesorado, consecuentemente, tiene el deber de "reciclarse". Se destaca el papel determinante que debe tener la formación del profesorado para un uso educativo de los materiales didácticos digitales (Rodríguez Rodríguez y Martínez Bonafé, 2016; Rodríguez Rodríguez y Rodríguez Regueira, 2016) .

\section{Propuestas de mejora}

Teniendo en cuenta las reflexiones anteriores se llega a la conclusión de que al profesorado entrevistado le gustaría seguir trabajando con E-DIXGAL en los próximos años, sin embargo, su satisfacción con el proyecto no es plena.

Existe una coincidencia absoluta entre las personas entrevistadas en el momento de indicar propuestas de mejora relacionadas con la organización de los contenidos y editoriales. A continuación, se consideran las mejoras de carácter general pertenecientes a gran parte de los/as entrevistados. 
- $\quad$ Mayor número de oferta editorial y contenidos contextualizados.

- Formación TIC del profesorado en función de los niveles, más centrada en metodologías participativas.

- Formación TIC del alumnado desde cursos inferiores con la finalidad de facilitar la adaptación al proyecto.

- Seguir trabajando en el incremento de la calidad de la conexión a la red tanto en áreas rurales como en áreas urbanas.

- Hacer un proyecto integral de introducción TIC.

- Crear una propuesta de actividades más interactivas, que favorezcan la adquisición de las competencias básicas.

- Añadir la posibilidad de que el alumnado pueda subir contenidos a la red para no tener que emplear otras redes ajenas.

- $\quad$ Promover una adecuada continuidad del proyecto en los IES.

Para finalizar, llama la atención que tan sólo uno de los ocho participantes añadiera en sus comentarios la posibilidad de que el alumnado pudiera subir contenido a la red a pesar de que en las entrevistas se abordaron todos los aspectos de la CD, incluido la creación de conocimiento. La continuidad de E-DIXGAL en la ESO también resulta interesante en el sentido de adoptar un modelo pedagógico que impregne todo el sistema educativo, sin fracturas, que permita el desarrollo integral de las personas.

\section{Discusión y conclusiones}

Los resultados obtenidos en el punto anterior demuestran que cada centro y cada maestro/a poseen unas características peculiares y únicas. Sin embargo, durante las entrevistas hemos observado varios puntos comunes a todos los participantes que han generado debate: por una parte, el proyecto E-DIXGAL, fruto de una idea insuficientemente desarrollada, tiene como consecuencia un desajuste entre el propio material y las necesidades o aspiraciones de los maestros; y por otra parte, se describe el deseo de un modelo pedagógico acorde con los tiempos, de corte constructivista, con materiales en consonancia con esa concepción de la enseñanza.

Relativo a esta visión actualizada de la educación en la cual el medio digital ocupa un amplio espacio, los resultados obtenidos indican que los docentes si reconocen su uso, cada vez mayor, entre los discentes. No obstante, se observa una contradicción entre ese reconocimiento y lo que sucede en sus aulas, los alumnos y alumnas apenas crean contenido propio (Mato-Vázquez y Álvarez-Seoane, 2019). Es decir, trabajan con la información para mejorar su comprensión (véase hacer una presentación en Powerpoint sobre un tema del libro digital), pero se elude la posibilidad de desarrollar el espíritu crítico.

Este hecho puede llevarnos a pensar en los porqués de esa aparente reticencia a la creación. Para explicarlo debemos tener en cuenta todas las categorías expuestas; desde la escasa formación en TICs, pasando por la disensión interna que viven los docentes cuando intentan cambiar su metodología hacia propuestas didácticas como los proyectos en red, e-portafolio o pedagogía inversa, entre otros. De hecho, conviene destacar las propuestas sobre el e-portafolio como el de Fraga Varela y Gewerc Barujel (2009), y sobre la pedagogía inversa, como el de Núñez Marín y Gutiérrez Porlán (2016). En ambas se visualiza el potencial de la metodología, el considerable esfuerzo que lleva planificar todas las sesiones y el tiempo como condición de mejora. 
Así es que durante las entrevistas se ha apreciado una clara percepción del tiempo como un bien escaso al tratar de trabajar desde la totalidad del currículo. Además, en algunas categorías los y las participantes tuvieron dificultades para comprender el significado de preguntas, como por ejemplo, las que involucran el término competencia digital. Entonces se descubre la importancia que tiene la coordinación entre los distintos agentes educativos -familias, profesorado, alumnado, consejerías, ministerios- para poder avanzar hacia una misma dirección de forma conjunta.

La otra cuestión que es reconocida por todos y todas las participantes es la visión de la editorial $A B$ como un PDF, un libro de texto que no posibilita modificar el temario. En particular, la valoración es negativa en todos los casos, se considera que responde a la pedagogía de la enseñanza por exposición y aprendizaje por recepción (Alonso Ferreiro, 2016; Area, 2011, 2015), con el añadido de actividades interactivas con retroalimentación inmediata que no proporcionan una explicación del error, de ser el caso. Esto coincide con las prácticas de aula observadas en la tesis doctoral de Alonso Ferreiro (2016), las cuales advierten del uso de estos medios para hacer lo que ya se hacía antes, que dificulta el desarrollo de la CD en el alumnado. Resultados semejantes se recogen en el trabajo sobre las visiones del alumnado sobre los materiales didácticos digitales en España (RegoAgraso, Marín Suelves, 2019).

Así, los paquetes de programas o materiales educativos informáticos de corte conductista siguen activos, aunque de forma disimulada, ya que incorporaron los nuevos elementos de la tecnología multimedia como por ejemplo los enlaces, material audiovisual o interacción retroalimentación directa humano-máquina que comentan todos/las los/las participantes de la investigación. El libro de texto digital no parece ayudar a romper con la idea de un circuito cerrado en el que se presupone que el libro determina en gran medida el desarrollo curricular del aula (Rodríguez Rodríguez y Martínez Bonafé, 2016). Por eso, tal y como afirman estos autores, resulta difícil encontrar en el libro impreso o el libro digital un tratamiento adecuado al discurso pedagógico renovador.

El libro digital sobre el que se articula el Proyecto E-DIXGAL ofrece la falsa seguridad de estar realizando correctamente la tarea escolar, cuyos contenidos se acercan a la objetividad de la concepción técnica de la enseñanza. Del mismo modo, actúa como limitador en el sentido de proveer contenidos cerrados, sin posibilidad de interpretación ni duda, cuyas actividades de pensar, reflexionar e investigar son más bien esporádicas y circunstanciales. Por lo tanto, su utilización exclusiva limita la práctica docente.

Como consecuencia, los entrevistados y entrevistadas siguen considerando los netbook como una herramienta de trabajo o un recurso de apoyo a la actividad formativa que se desarrolla en el aula desde antes. Sin embargo, en el otro extremo podría encontrarse la concepción del modelo $1: 1$ como un ecosistema social y cultural que se puede emplear para que los estudiantes aprendan mediante la creación, producción y construcción de saber (Area, 2015).

En definitiva, tras los resultados obtenidos se puede afirmar que sí existe un cambio en la visión del profesorado, relativamente lento, teórico y poco presente en la práctica. Esta situación pone de manifiesto que el profesorado ni está totalmente preparado para integrar las TIC diariamente ni cuenta con el apoyo necesario desde la comunidad y la propia Administración para llevar a cabo ese cambio. La tecnología se emplea y se trata de planificar la programación didáctica con ella, pero no se va más allá de actividades de práctica y ejercitación (Alonso Ferreiro, 2016; Gewerc Barujel y Pernas Morado, 1998), a no ser que ya se hubiera adoptado otra metodología de aula con anterioridad, como en el caso del CEIP RC que usa E-DIXGAL como una enciclopedia virtual.

Por lo que se refiere al futuro del proyecto, el profesorado también muestra desconfianza hacia la continuidad del mismo, que en ocasiones se ve truncado en la etapa de la Educación Secundaria Obligatoria (ESO). Es decir, no se atiende a todo el alumnado en edad obligatoria, sino que el proyecto está destinado a $5^{\circ}$ y $6^{\circ}$ de EP y $1^{\circ}$ y $2^{\circ}$ de la ESO. 
Por último, sólo queda decir que este trabajo permite obtener una visión bastante específica de la opinión del profesorado de Educación Primaria que trabaja con E-DIXGAL. Aun así creemos necesario seguir avanzando en esta línea de investigación dada la importancia del profesorado como motor de cambio, esencial en el desarrollo integral de los alumnos y alumnas. La evaluación del proyecto, la repercusión de las TIC, la brecha digital, la Competencia Digital, las nuevas vías de formación, las prácticas educativas innovadoras... son cada una de ellas una parte fundamental de los desafíos en la educación del siglo XXI.

\section{Referencias}

Adell, J. (1997). Tendencias en educación en la sociedad de las tecnologías de la información. Revista Electrónica de Tecnología Educativa, no 7. Recuperado de: http://www.edutec.es/revista/index.php/edutec-e/article/view/570/299

Alonso Ferreiro, A. (2016). Competencia Digital y escuela. Estudio de caso etnográfico en dos CEIP de Galicia. [Tesis Doctoral]. Santiago de Compostela: Universidad de Santiago de Compostela.

AMTEGA (2014). Proxecto Abalar Balance. Recuperado de: http://amtega.xunta.gal/documentos/Balance_abalar_2014.html

Area Moreira, M. (2011). Los efectos del modelo 1:1 en el cambio educativo en las escuelas. Evidencias y desafíos para las políticas iberoamericanas. Recuperado de: http://webcache.googleusercontent.com/search?q=cache: hLZICemVJAEJ:rieoei.org/rie56a02.pdf+\&cd=4 $\& h l=e s \& c t=c l n k \& g l=e s$

Area Moreira, M. (2015). Reconstruir el discurso pedagógico para la escuela de la sociedad digital. En Juan de Pablos Pons (coord.). Los centros educativos ante el desafío de las tecnologías digitales. Madrid: La muralla S.A.

Area Moreira, M., Cano, C. A., Gorospe, J. M. C., Pérez, M. E. D. M., Pons, J. D. P., Labra, J. P., ...y ValverdeBerrocoso, J. (2014). Las políticas educativas TIC en España después del Programa Escuela 2.0: las tendencias que emergen/ ICT education policies in Spain after School Program 2.0: Emerging Trends. Revista Latinoamericana de Tecnología Educativa-RELATEC, vol.13, n02, pp.11-33. Recuperado de: http://relatec.unex.es/article/view/1473

Barroso Jerez, C. (2013). Sociedad del conocimiento y entorno digital. Teoría de la Educación: Educación y Cultura en la Sociedad de la información (TESI), no 14, pp.61.86. Recuperado de: https://dialnet.unirioja.es/servlet/articulo?codigo $=4509593$

Bringué Sala, X., Sádaba Chalezquer, C., y Artopoulos, A. (2014). La generación interactiva en Argentina: niños y adolescentes ante las pantallas. Education Policy Analysis Archives/Archivos Analíticos de Políticas Educativas, 22, 1-19.

Cabero Almenara, J. (2015). Reflexiones educativas sobre las tecnologías de la información y la comunicación (TIC). CEF, $\mathrm{n}^{0} 1, \quad$ pp.19-27. Recuperado de: https://idus.us.es/xmlui/bitstream/handle/11441/32285/Reflexiones_educativas_sobre_las_Tecnolo.pdf? sequence $=1$ \&isAllowed $=y$

Castro Rodríguez, Ma M., Marín Suelves, D. y Sáiz Fernández, H. (2019). Competencia digital e inclusión educativa. Visiones de profesorado, alumnado y familias. RED. Revista de Educación a Distancia, 61.

Decreto 105/2014, do 4 de setembro, polo que se establece o currículo de educación primaria na Comunidade Autónoma de Galicia.

Fernández Morante, M.C. y Cebreiro López, B. (2003). La integración de los medios y nuevas tecnologías en los centros y prácticas docentes. Pixel Bit, Revista de Medios y Educación, n020. Recuperado de: http://acdc.sav.us.es/ojs/index.php/pixelbit/article/view/708/587

Fernández de la Iglesia, J.C., Fernández Morante, M.C. y Cebreiro López, B. (2016). Competencias en TIC del profesorado en Galicia: variables que inciden en las necesidades formativas. Innovación Educativa, n²6, pp.215-231. Recuperado de: http://www.usc.es/revistas/index.php/ie/article/viewFile/3256/3927 
Fraga Varela, F. y Alonso Ferreiro, A. (2016). Presencia del libro de texto digital en Galicia: una mirada estadístico-geográfica del proyecto E-DIXGAL. Profesorado. Revista de currículum y formación del profesorado Recuperado de: http://digibug.ugr.es/bitstream/10481/42554/1/rev201ART6.pdf

Fraga Varela, F. y Duarte Fernández, A. (2015). La perspectiva de las familias de un centro educativo digital con modelo 1 a 1: implantación del Proyecto E-DIXGAL como desarrollo del Plan Escuela 2.0 y el Proyecto Abalar en Galicia. Innovación Educativa, no 25, pp. 309-325. Recuperado de: http://www.usc.es/revistas/index.php/ie/article/view/2826

Fraga Varela, F. y Gewerc Barujel, A. (2009). E-portafolios. La búsqueda de un software coherente con la propuesta de enseñanza. RED, Revista de Educación a Distancia. Recuperado de: http://www.um.es/ead/red/M8/usc.pdf

Gewerc Barujel, A. y Pernas Morado, E. (1998). Los usos del ordenador en el aula: análisis de las observaciones de los alumnos/as de magisterio en prácticas. Innovación Educativa, n08, pp.295-305. Recuperado de: https://minerva.usc.es/xmlui/bitstream/handle/10347/5235/pg_297307_inneduc8.pdf?sequence $=1$ \&isAllowed $=y$

Martínez Bonafé, J. (2008). Los libros de texto como práctica discursiva. Revista de Asociación de la Sociología de la Educación (RASE). Recuperado de: https://dialnet.unirioja.es/servlet/articulo?codigo=2793153

Mato-Vázquez, D. y Álvarez-Seoane, D. (2019). La implementación de TIC y MDD en la práctica docente de Educación Primaria. Campus Virtuales, 8 (2), 73-84.

Montero Mesa, Ma L., Gewerc Barujel, A. (Coords) (2013). Una historia, cuatro historias: acompañar proyectos de innovación educativa con las TIC. Barcelona: Graó.

Núñez Marín, A. y Gutiérrez Porlán, I. (2016). Flipped learning para el aprendizaje del inglés en Educación Primaria. EDUTEC, Revista Electrónica de Tecnología Educativa, nº56. Recuperado de: http://www.edutec.es/revista/index.php/edutece/article/view/654/Edutec_n56_Nu\%C3\%B1ez_Gutierrez

Peirats Chacón, J., Gallardo Fernández, I. M., San Martín Alonso, A. y Cortés Mollá, S. (2015). Los contenidos curriculares digitalizados, voces y silencios en el ámbito editorial. Educatio Siglo XXI, vol. 33 , no 3. Recuperado de: http://revistas.um.es/educatio/article/view/240801/184461

Pérez Sanz, A. (2011). Escuela 20. Educación para el mundo digital. Recuperado de: http://www.injuve.es/sites/default/files/RJ92-06.pdf

Rego-Agraso, L. y Marín Suelves. D. (2019). Las visiones del alumnado sobre los materiales didácticos digitales. Educar em Revista, 35,77, 79-94.

Rodríguez Machado, E. (2015). Aproximación Teórica a Edixgal. Libro digital de Educación Física. Sportis: Revista Técnico-Científica del Deporte Escolar, Educación Física y Psicomotricidad, 1(1), 2-15. Recuperado de: http://revistas.udc.es/index.php/SPORTIS/article/view/1398/807

Rodríguez Machado, E., Veiga, J.E. y González Sanmamed, M. (2015). O Proxecto Abalar e o Libro Dixital en Galicia (Edixgal). Revista de Estudios e Investigación en Psicología $\quad$ y Educación, $\quad$ no 13. Recuperado de: http://revistas.udc.es/index.php/reipe/article/view/592/pdf_395

Rodríguez Rodríguez, J., Braga García, T. y Bruillard, E. (2019). (coords). IARTEM 1991-2016: 25 Years developing textbook and educational media research. Kongsberg: IARTEM.

Rodríguez Rodríguez, J. y Martínez Bonafé, J. (2016). Libros de texto y control del curriculum en el contexto de la sociedad digital. Cadernos CEDES, vol.36, no100. Recuperado de: http://www.scielo.br/pdf/ccedes/v36n100/1678-7110-ccedes-36-100-00319.pdf

Rodríguez Rodríguez y Rodríguez Regueira (2016). Revisión de la investigación publicada sobre el libro de texto digital en revistas, publicaciones y congresos internacionales de referencia. Profesorado. Revista de curriculum y formación del profesorado, 20, 1, 9-31.

Sanabria Mesa, A. L. Álvarez Núñez, Q. y Peirats Chacón, J. (2017). Las políticas educativas en la producción y distribución de materiales didácticos digitales. Relatec, 16 (2), 63-77.

Sanmartín Alonso, Á. y Peirats Chacón, J. (2018). Controversias en la transición del libro de texto en papel y electrónico a los contenidos digitales. RED. Revista de Educación a Distancia, 56, Art. 5. 